\title{
Beam-energy-spread minimization using cell-timing optimization
}

\author{
C. R. Rose, C. Ekdahl, and M. Schulze \\ Los Alamos National Laboratory, P.O. Box 1663, Los Alamos, New Mexico 87545, USA
}

(Received 12 September 2011; published 11 April 2012)

\begin{abstract}
Beam energy spread, and related beam motion, increase the difficulty in tuning for multipulse radiographic experiments at the dual-axis radiographic hydrodynamic test facility's axis-II linear induction accelerator (LIA). In this article, we describe an optimization method to reduce the energy spread by adjusting the timing of the cell voltages (both unloaded and loaded), either advancing or retarding, such that the injector voltage and summed cell voltages in the LIA result in a flatter energy profile. We developed a nonlinear optimization routine which accepts as inputs the 74 cell-voltage, injector voltage, and beam current waveforms. It optimizes cell timing per user-selected groups of cells and outputs timing adjustments, one for each of the selected groups. To verify the theory, we acquired and present data for both unloaded and loaded cell-timing optimizations. For the unloaded cells, the preoptimization baseline energy spread was reduced by $34 \%$ and $31 \%$ for two shots as compared to baseline. For the loaded-cell case, the measured energy spread was reduced by $49 \%$ compared to baseline.

DOI: 10.1103/PhysRevSTAB.15.040403

PACS numbers: 29.20.Ej, 41.75.Fr
\end{abstract}

\section{INTRODUCTION}

The dual-axis radiographic hydrodynamic test (DARHT) facility at Los Alamos National Laboratory (LANL) uses high-energy $\mathrm{x}$ rays from two perpendicular axes to perform multipulse radiographic experiments. There is considerable information about DARHT in the literature. Thus, by way of brief summary, both axis-I and axis-II are linear induction accelerators (LIAs) with 60-ns and 1.6- $\mu \mathrm{s}$ pulse widths, respectively [1-5]. Figure 1 shows a simplified diagram of axis-II consisting of the injector, accelerating cells, kicker, and target. Electron particle acceleration is accomplished by the injector and 74 induction cells [6-12]. Pulse forming networks (PFNs) provide power (voltage and current) to the accelerating cells [13-15]. Normally, the beam is bent and collected at the beam dump. When performing experiments, the kicker chops the beam and sends beam packets or bunches down the transport line to the $\mathrm{x}$-ray converter (target) [16-18]. X rays illuminate the device under test, and special cameras record the images.

Beam motion, beam-energy spread, and the ability to achieve multipulse minimal spot size are linked. Beam motion is detrimental to the multiple-pulse radiography performed by the axis-II LIA. Radiographic source spots are blurred by time integration of motion having many periods during a micropulse width. Motion with period greater than a micropulse width causes spot-to-spot displacements, and distorts the last spots in a multipulse

Published by the American Physical Society under the terms of the Creative Commons Attribution 3.0 License. Further distribution of this work must maintain attribution to the author(s) and the published article's title, journal citation, and DOI. sequence due to asymmetric erosion of target material by the earlier spots.

A leading cause of low-frequency, beam motion in axisII is time-dependent variation of beam energy [19]. This results from beam deflection by random dipole fields in the LIA caused by misalignment of the focusing solenoids and winding errors [20], as well as any dipole fields applied to center the beam. The deflection of the beam in these transverse fields is inversely proportional to the momentum, so any temporal variation in energy causes beam motion. Since this happens in a strong axial focusing field, the motion is mostly in the form of a rotation of centroid position on the surface of a flux tube [19].

The correlation between beam motion and energy variation is obvious in Fig. 2, which compares the energy variation with motion in the vertical direction. Clearly, minimizing the beam-energy variation is a strategy to be pursued in our efforts to reduce beam motion with the objective of improving multipulse radiography.

Ideally, the sum of the cell voltages plus the injector voltage is a flat temporal profile with minimal variation over the duration of the pulse. Induction-cell voltage profiles are normally made flat by tuning the output impedance of the PFNs to match the impedance of the cells when beam is present. Substantial work was done to tune the PFNs with the cells and very good flattops with minimal energy spread were obtained [14]. In the DARHT project, the decision was made to tune the PFNs with a nominal output impedance such that when any PFN is connected to any cell, the combination would yield an acceptable flattop. Alternatively, to tune each cell with its respective driving PFN, as a pair, would have been not only expensive at the time of design and commissioning, but it would have been prohibitively time consuming once the facility became operational when a cell or PFN needs to be replaced. 


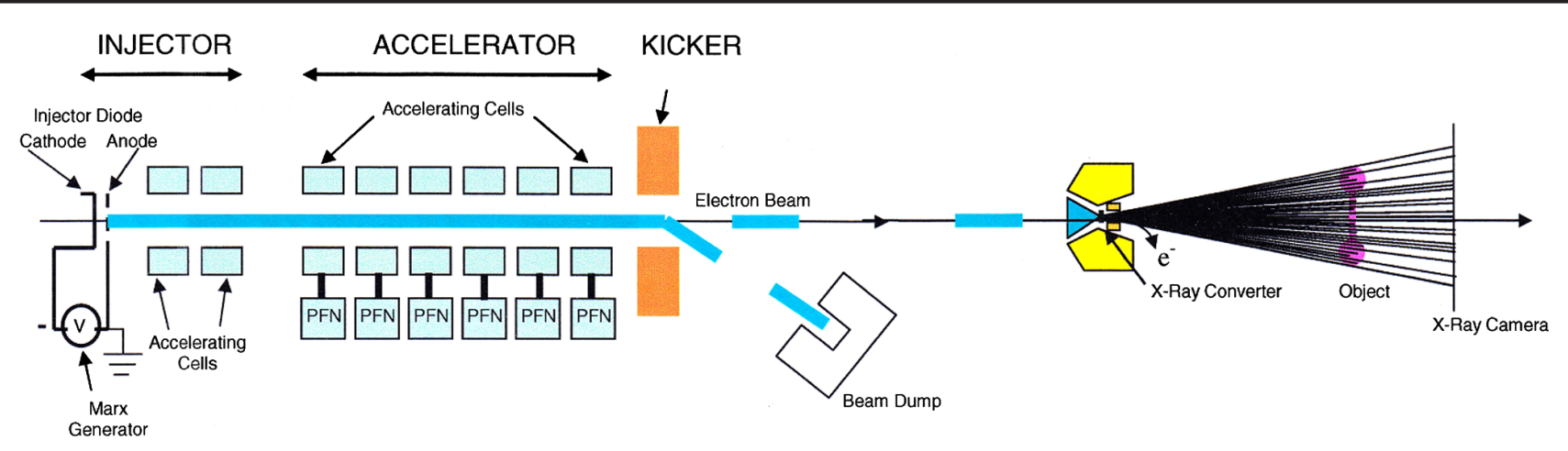

FIG. 1. DARHT axis-II accelerator overview [13].

When commissioned, axis-II met design and performance requirements for beam energy, beam current, energy spread ( \pm 0.5 percent) and radiography [6]. Since then, as a result of changes in operational settings such as lower injector voltage, lower cell voltages (225 to $200 \mathrm{kV})$ and beam current ( 1.85 to $1.65 \mathrm{kA}$ ), the energy spread is about \pm 1.2 percent.

At the present time, there are at least a few options to reduce energy spread. One is to adjust the injector voltage flattop which is very difficult to do. Another is to retune each PFN/cell combination in situ which is contrary to the original design and operational model. The 74 PFNs could also be retuned to better match present operating conditions. There is a need to be able to minimize beam energy spread in a timely and cost-effective manner taking into account existing constraints and operational parameters while allowing for operational changes in the future.
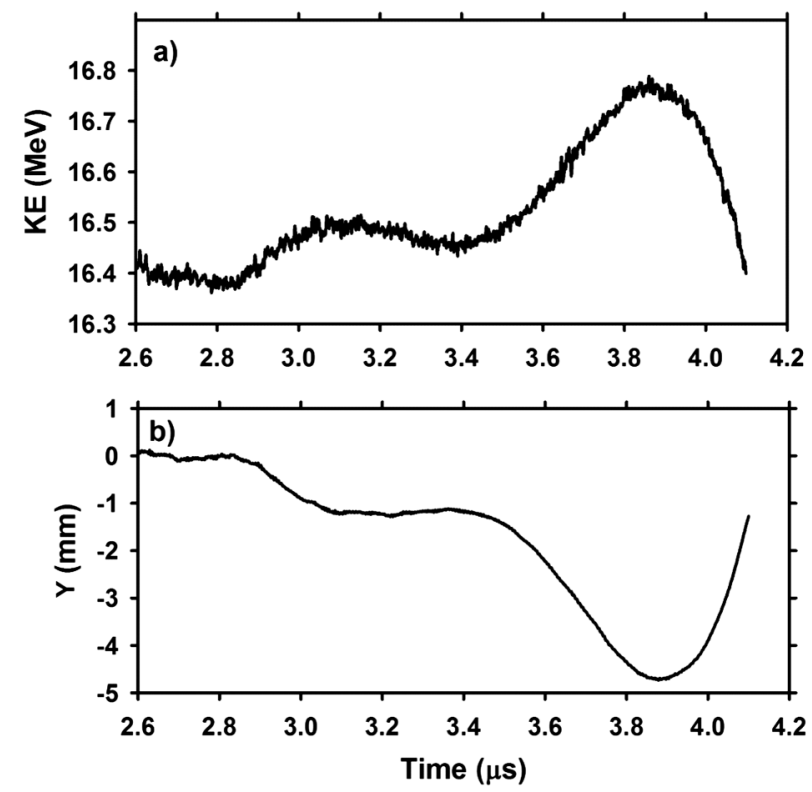

FIG. 2. Comparison of (a) beam energy as function of time with (b) beam vertical position at accelerator exit showing the correlation between the two.
This paper describes a theory and methodology to minimize beam energy spread using optimized cell timing. It is an entirely new approach, not previously tried on any LIA. The underlying premise is that there exists a set of optimal time advances or delays, one for each of the cells such that when implemented, the summation of the injector and cell voltages over a defined flattop window has lower peak-topeak deviation or lower energy spread than the same flattop measurement without cell time adjustment. In short, the peaks of some cell voltages will fill in the valleys of others making an overall flatter result with less temporal variation.

Following this introduction, Sec. II describes the optimization method and model output used to adjust cell timing. In Sec. III, the optimization method is applied to unloaded cell voltages, i.e., without beam. A baseline energy spread is presented, followed by predicted and measured values. A sensitivity analysis shows that the method is robust as regards systemic timing jitter. Section IV applies the optimization method to the loaded-cell case, i.e., with beam, and presents baseline data, predicted and measured results. Section V is a summary and concludes with a brief description of future work.

\section{OPTIMIZATION METHOD}

On axis-II, one timing or trigger signal nominally drives a group of four PFNs. Additionally, there is one group of three PFNs, and three individually triggered PFNs. Clearly, the preferred method would be to find optimal timing solutions for each of the 74 individual cell/PFNs; however, to do this would require a significant infrastructure upgrade and the replacement of the 21 operating four-channeltrigger units with 74 single-channel-trigger units. If we are able to significantly reduce the energy spread by varying only 21 groups, or some subset of them, it is highly probable that even more reduction in energy spread could be achieved by adjusting the 74 individually.

We developed a nonlinear optimization routine using MATLAB [21] that uses as inputs the injector voltage waveform (loaded case), the beam current waveform (loaded case), and the voltage vs time waveforms for each of the 
74 cells (loaded and unloaded). For the unloaded case, the routine uses the voltage profiles directly, and for the loaded-cell case, it uses the input data and synthesizes cell voltages that can be time shifted to reflect how they would appear as they are advanced or retarded with respect to the beam showing the effects of voltage perturbations in relation to the timing of the beam. Additionally, the user can select which trigger units are fixed in time, and which units the optimizer will adjust. For loaded models, cell volt-second saturation effects are also included. When executed, the routine adjusts timing of the selected groups to minimize a cost function. Outputs from the routine consist of 1 to 21 time adjustments, one for each of the selected four-channel-trigger units, calculated relative to the timing of the input data sets. The optimization model stops when a predetermined error tolerance has been met. The cost function is the minimum of normalized beamenergy spread $\hat{S}_{\mathrm{BE}}$, as a percentage, stated as

$$
\min \left(\hat{S}_{\mathrm{BE}}\right)
$$

where

$$
\begin{gathered}
\hat{S}_{\mathrm{BE}}=\frac{\Delta}{2 \bar{E}} \times 100 \\
\Delta=\max (|\tilde{V}|)-\min (|\tilde{V}|)
\end{gathered}
$$

with midlevel beam energy,

$$
\bar{E}=\frac{\max (|\tilde{V}|)+\min (|\tilde{V}|)}{2}
$$

and

$$
\tilde{V}=V_{\mathrm{inj}}\left[t_{0}, t_{1}\right]+\sum_{n=1}^{N} V_{n}\left[t_{0}, t_{1}\right]
$$

where $N$ is the number of cell-voltage waveforms, $V_{\text {inj }}$ is the injector voltage, $V_{n}$ represents the voltage waveform from the $n$th cell, and $t_{0}$ and $t_{1}$ are the beginning and end times of the flattop measurement window. Note that $\tilde{V}, \Delta$, and $\bar{E}$ are in MV.

The solution space for the timing optimization has multiple local minima. To compensate for this, a random restart technique of seeding the optimizer with uniformdistribution random starting values, $U[-250 \mathrm{~ns}, 250 \mathrm{~ns}]$, for each of the trigger groups was employed, then the $\hat{S}_{\mathrm{BE}}$ results were evaluated for the lowest value and the corresponding timing adjustments were selected as the global minimum optimum solution given the constraints. The optimization algorithm is moderately computationally expensive for the loaded-cell simulations, allowing random restarts of up to 2500 iterations in less than 24 hours running on a xeon-class workstation.

\section{UNLOADED CELLS}

In this section, the baseline energy spread is measured using a sample data set and (1)-(5) with $V_{\text {inj }}$ in (5) set to zero. Then, using the same data set which was used to compute the baseline, the optimizer determines appropriate timing adjustments to yield the best-case reducedenergy spread. We also call this the predicted minimum energy spread, or simply predicted energy spread. The predicted energy spread is then compared with two shots timed per the optimizer-derived solution set. Sensitivity analysis results are shown and compared to the predicted and measured temporal energy profiles.

\section{A. Baseline}

As a first step to verify the underlying concepts and optimization method, we used unloaded cells because they retain the same voltage vs time profile as their timing is either advanced or retarded. This assumption, though not accurate when beam is present, simplifies the preliminary models and simulations while demonstrating the feasibility of the underlying research approach.

The unloaded baseline energy spread was computed using nominal timing from shot 14753. Figure 3 shows the 74 voltage waveforms including markers for the flattop window spanning from $t_{0}=2.5 \mu \mathrm{s}$ to $t_{1}=4.05 \mu \mathrm{s}$. Obviously, other measurement windows will yield different energy spreads. The objective is to widen the window as much as possible while yielding acceptable spread. The window used in these simulations is close to where the accelerator is normally operated.

The baseline beam-energy temporal profile is the top trace shown in Fig. 4. Note the large energy excursion at about $3.8 \mu \mathrm{s}$. We should mention that the predicted and measured results have been added to this figure for easy comparison and will be discussed in later sections.

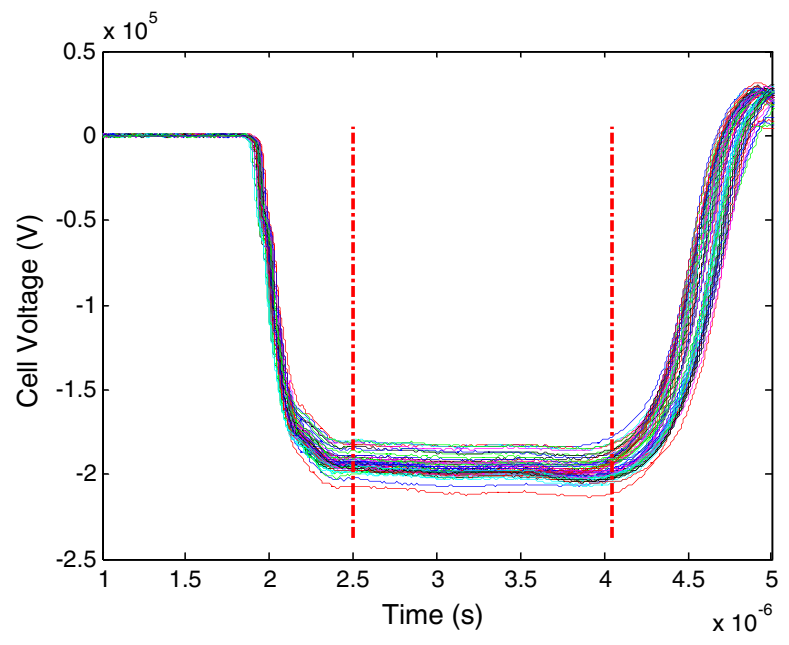

FIG. 3. Voltage waveforms for 74 cells on axis-II for shot 14753. The analysis window is between 2.5 and $4.05 \mu \mathrm{s}$. 


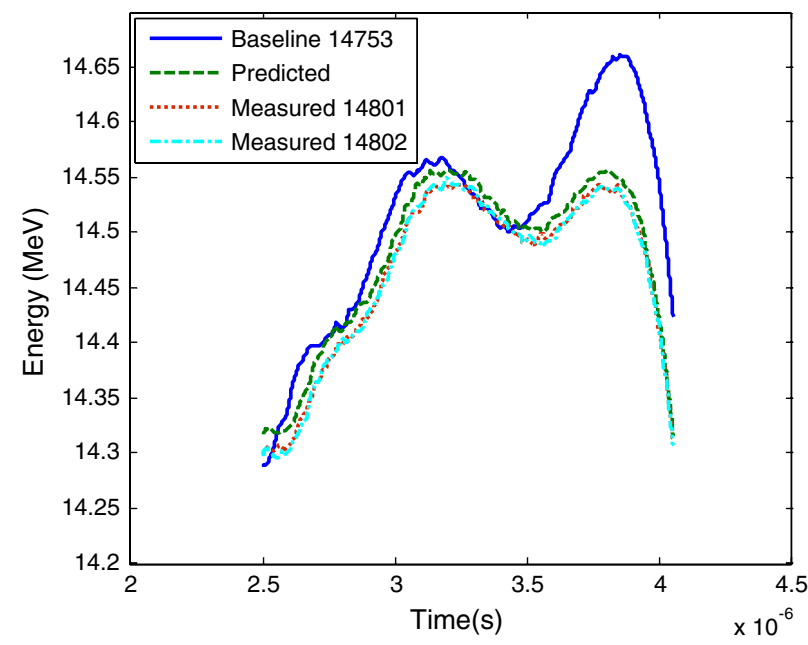

FIG. 4. Comparison of energy spread for baseline, predicted, and shots 14801 and 14802 .

Shown in Fig. 4, the baseline energy spread is measured as \pm 1.286 percent, and the midlevel energy is $14.475 \mathrm{MeV}$. The voltage waveform sampling period is $2 \mathrm{~ns}$, and to reduce noise, the waveforms have been smoothed with a 7-point, forward-backward, moving-average filter. Note that the number of significant digits in the midlevel energy are too many for practical systems. However, we are showing that amount of implied accuracy for easy comparison to the waveforms that follow. Actual systems will be valid to no more than four, and maybe three significant digits.

\section{B. Predicted}

The predicted, minimal energy spread was found by using the waveform data from 14753 and inputting it to the optimizer routine. The data from 14753 incorporated both nominal timing information and voltage vs time profiles for each of the cells. To compute the optimal time shifts, 10000 random restart iterations were executed, with the best solution set obtained at iteration 4520. Figure 5 shows the cell-voltage waveforms of 14753 time shifted or adjusted by the optimizer for minimal spread. The bestcase (predicted) energy spread of $\pm 0.831 \%$ is shown in Fig. 4 and can be compared to the baseline temporal profile. We should mention that the predicted curve is the energy spread profile that we would expect to obtain if axis-II could be operated with the exact timing as determined by the optimizer. In this case, the spread has been reduced by $35 \%$ compared to baseline and the midlevel energy is slightly decreased to $14.436 \mathrm{MeV}$.

The timing adjustments for the 21 trigger units varied from +119 to $-142 \mathrm{~ns}$ with one at $-618 \mathrm{~ns}$. Negative values advance the timing, and positive values retard it.

\section{Measured}

After adjusting the timing of the trigger units, two shots (without beam) were executed-14801 and 14802-to

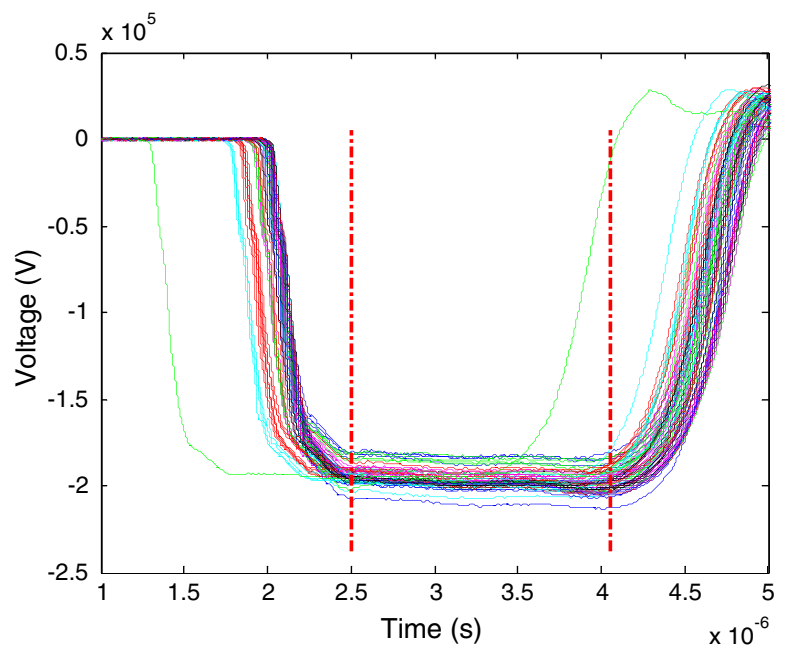

FIG. 5. Cell-voltage waveforms for 74 cells on axis-II for shot 14753 based on optimized timing for 21 groups. The analysis window is shown between 2.5 and $4.05 \mu \mathrm{s}$.

observe and measure the resulting energy temporal profile. Figure 6 shows the measured cell voltages for 14801. The resulting energy spread of \pm 0.847 percent is shown in both Figs. 4 and 8 for easy comparison. The midlevel energy is $14.422 \mathrm{MeV}$.

There is a small energy offset between the predicted and measured energy profiles of about $0.12 \%$ which can be attributed to tolerances on high-voltage power supply settings and instrumentation.

Cell voltages for shot 14802 , using the same timing settings as 14801, are shown in Fig. 7. The energy spread for 14802, shown in Figs. 4 and 8, is $\pm 0.881 \%$ and the midlevel energy spread is $14.423 \mathrm{MeV}$.

Moreover, the percentage increase in energy spread from the predicted value is $1.9 \%$ and $6 \%$ for shots 14801 and

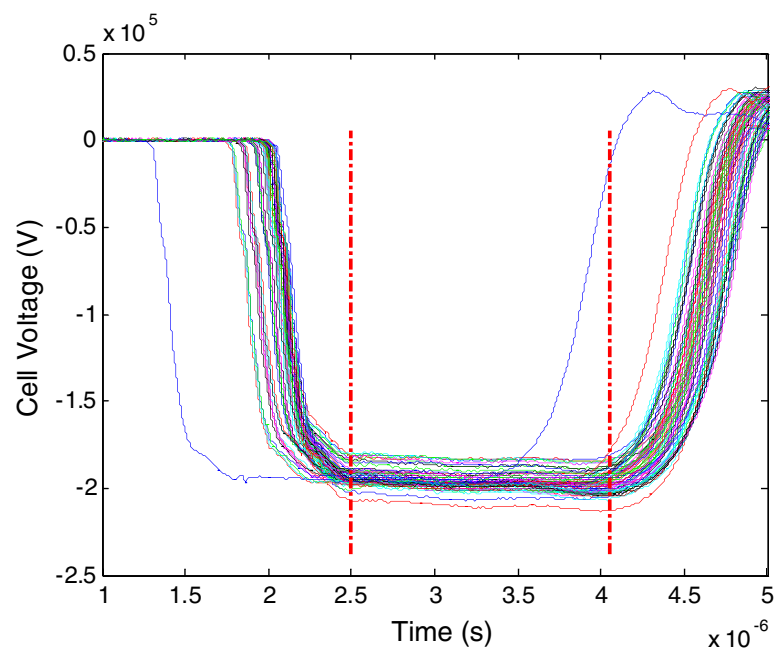

FIG. 6. Shot 14801 showing all 74 voltage waveforms with optimized timing. Resulting energy spread is \pm 0.847 percent energy spread which is slightly larger than the predicted value. 


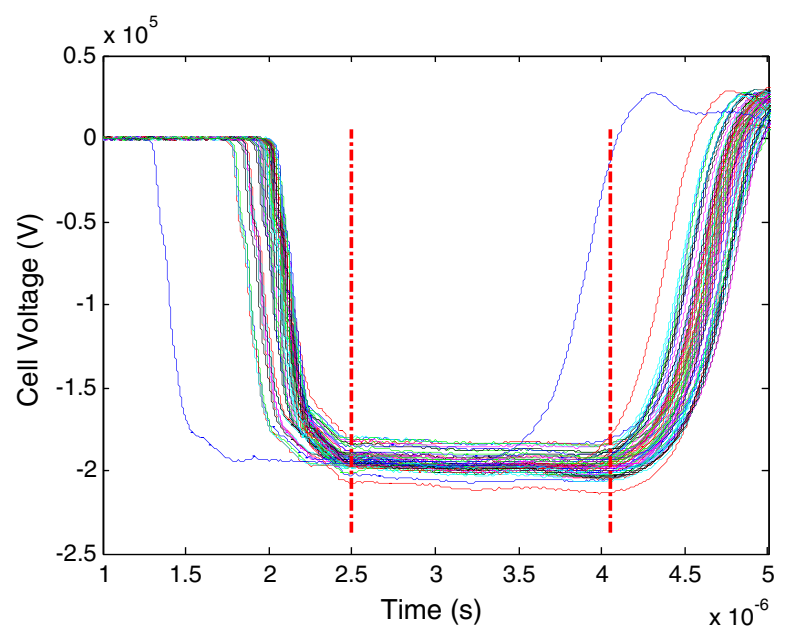

FIG. 7. Shot 14802 showing 74 voltage waveforms with optimized timing. Resulting energy spread is \pm 0.881 percent.

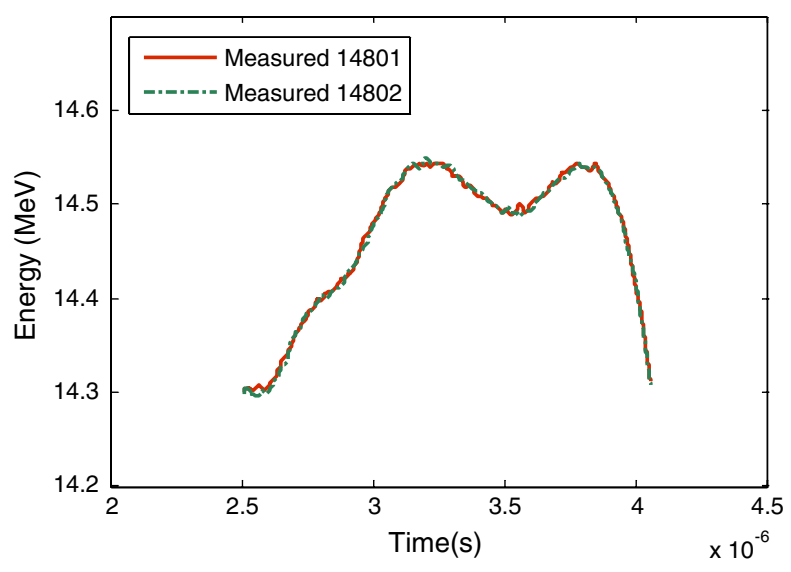

FIG. 8. Comparison of energy spread for shots 14801 and 14802 showing close agreement.

14802, respectively, and the improvements compared to baseline are $-34 \%$ and $-31 \%$. Thus, they both have significantly less spread than baseline even with some systemic variability.

Figure 8 shows close agreement between measured energy spreads. In fact, although shot 14802 has a higher spread of $\pm 0.881 \%$, it is only $4 \%$ larger than the measured spread of shot 14801 which can be attributed to perturbations near 2.6 and $3.2 \mu \mathrm{s}$. The remaining portions of the two waveforms overlay almost exactly.

We attribute the variation in energy spread between the predicted and the test shots, 14801 and 14802 (see Fig. 4), to timing jitter on the cell voltages. This jitter is due to variations within the trigger units and the PFNs themselves. A histogram of measured, 1- $\sigma$ (1 standard deviation) cell-timing jitter is shown in Fig. 9 using data taken from June 2011. Typical, measured-jitter values are in the range of 2 to $16 \mathrm{~ns}$ depending on the PFN/cell. The average, or centroid, of the histogram, is just over $6 \mathrm{~ns}$.

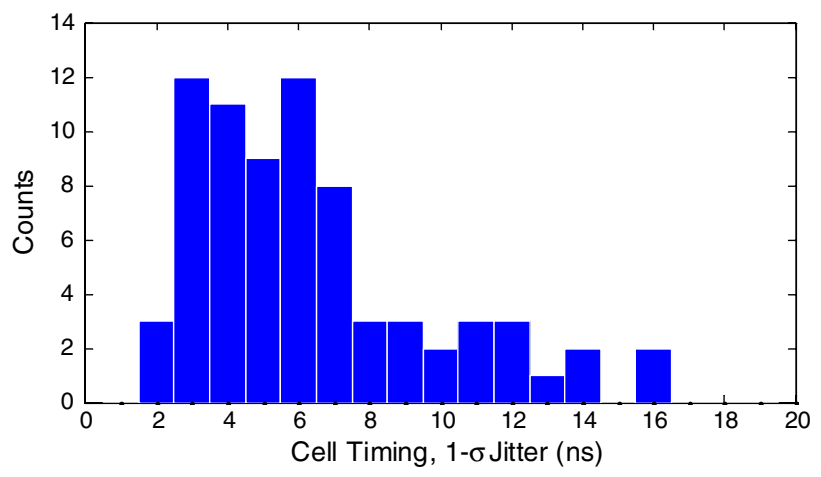

FIG. 9. Histogram showing the distribution of cell-voltagetiming jitter (1- $\sigma, 1$ standard deviation). Data are from June 2011.

This is the value that, if used for all the cells, would yield equivalent results as compared to cells that had different, and individual, random jitter probability density functions.

\section{Sensitivity analysis}

An essential aspect of any optimization method is the sensitivity of the predicted, reduced-energy spread to cellvoltage-timing jitter. This timing jitter, illustrated in the histogram of Fig. 9, is intrinsic to the accelerator, and its results are reflected in the data from shots 14801 and 14802. As the cell voltage timing is varied, the voltage profile in the measurement window also changes, some portions higher or lower. There is also a small voltage amplitude variation from shot to shot, measured at identical time points, but we view this as negligible in comparison to the voltage fluctuations due to timing variations.

To better quantify the change in energy spread vs jitter, a sensitivity analysis using a Monte Carlo (MC) technique was executed. The predicted, model output of Fig. 5, with perfect timing, was used as input to the MC simulator which added random, Gaussian timing jitter, $N[0, \sigma]$, to each of the 74 cell-voltage waveforms, and at each iteration, the simulator computed the energy spread using (2)-(5). Standard deviation values of 0.5 through $20.0 \mathrm{~ns}$ were used, each zero mean. These values along with the MC-simulator-derived maximum-energy spread are shown in Table I. Note that with 0.0-ns jitter, the output energy spread remains the same at $\pm 0.831 \%$ as expected.

After 250000 iterations at each jitter value, the maximum-energy spread was determined and used as the worst-case scenario for that particular value. An important point to recall is that these are the worst-case energy spreads based on the simulations, and any actual energy spread will likely be less. After all, these spreads are based on 250000 solution sets, looking for the worst one among them, instead of just one or two shots. As a result, the spread vs jitter values in Table I are higher than actual performance would predict if all cells had similar timing jitter distributions. 
TABLE I. Listing of Monte Carlo simulated worst-case energy spread vs standard deviation of cell-voltage timing jitter.

\begin{tabular}{lc}
\hline \hline Jitter, $1-\sigma(\mathrm{ns})$ & \pm Worst-case energy spread $(\%)$ \\
\hline 0.0 & 0.831 \\
0.5 & 0.836 \\
1.0 & 0.841 \\
2.0 & 0.851 \\
3.0 & 0.860 \\
4.0 & 0.868 \\
5.0 & 0.880 \\
6.0 & 0.897 \\
7.5 & 0.900 \\
10.0 & 0.929 \\
15.0 & 0.962 \\
20.0 & 0.997 \\
\hline \hline
\end{tabular}

Using the estimated centroid from Fig. 9, i.e., a distributed jitter of $6 \mathrm{~ns}$ on all 74 cells, the worst-case energy spread was simulated to be $\pm 0.897 \%$ which effectively set maximum limits on actual shots. Note that the measured energy spread of shot 14801 was $\pm 0.847 \%$, and shot 14802 was $\pm 0.881 \%$, both lower than the simulated limits.

\section{LOADED CELLS}

In this section, the baseline data, predictions, and measured results for the loaded-cell experiments are discussed. Because the cells are loaded, the optimization routine synthesizes voltages for the cells that will be time adjusted and uses nominal empirical voltage waveform data for the nonshifted cells. Additionally, to minimize risk to the accelerator while the models are being verified, we limited timing optimization to the last three trigger units or cells, 72, 73, and 74 (trigger chassis 19, 20, 21), and kept the timing of cells 1-71 fixed (trigger chassis $1-18)$.

\section{A. Baseline}

The baseline loaded-cell energy spread was computed using data from shot 16729 . Figure 10 shows the baseline injector voltage. The peak is $-2 \mathrm{MV}$, and the pulse is truncated at $4.15 \mu$ s via a crowbar system. The injector voltage is supplied by a high-voltage Marx generator charged to $\pm 31 \mathrm{kV}$. The beam current for this pulse is shown in Fig. 11, and has a peak value of $1.66 \mathrm{kA}$. Figure 12 shows the loaded-cell voltages for all 74 cells. Note the perturbation in cell voltage near the end of the main pulse, about $4.15 \mu \mathrm{s}$, when the beam current goes to zero and the cells become unloaded.

The top trace in Fig. 13 shows the baseline energy spread. Its deviation is $\pm 1.126 \%$, and the measurement window is from 2.5 to $4.0 \mu$ s. Additionally, in Fig. 13, the predicted and measured temporal energy spread curves are

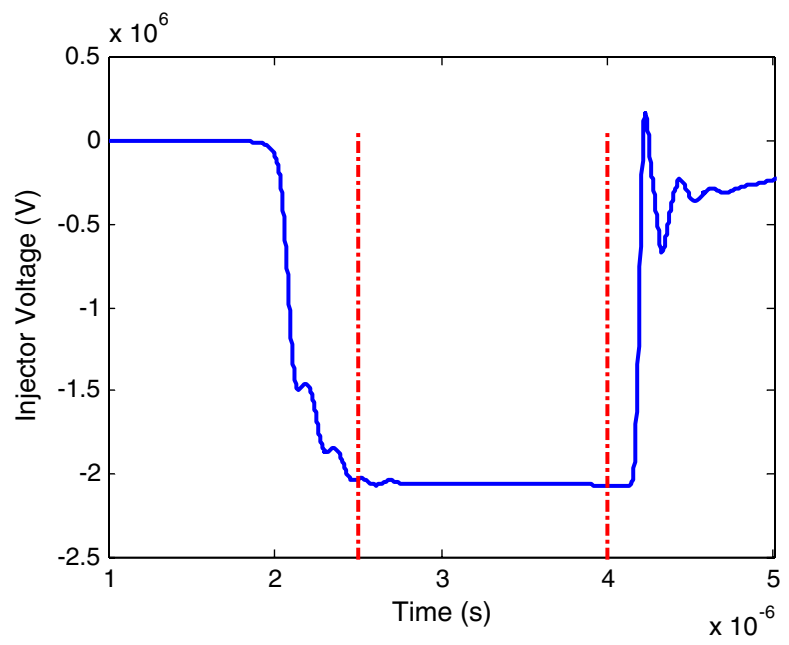

FIG. 10. Baseline configuration injector voltage (shot 16729). The voltage is truncated at $4.15 \mu \mathrm{s}$ by an external crowbar system. Flattop measurement window is from 2.5 to $4.0 \mu \mathrm{s}$.

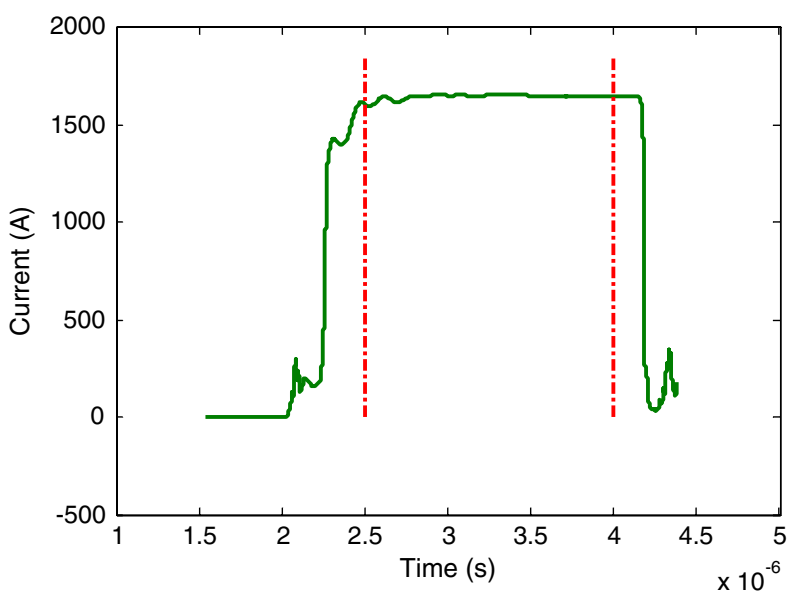

FIG. 11. Plot of beam current versus time. The peak value is $1.66 \mathrm{kA}$. The flattop measurement window is from 2.5 to $4.0 \mu \mathrm{s}$.

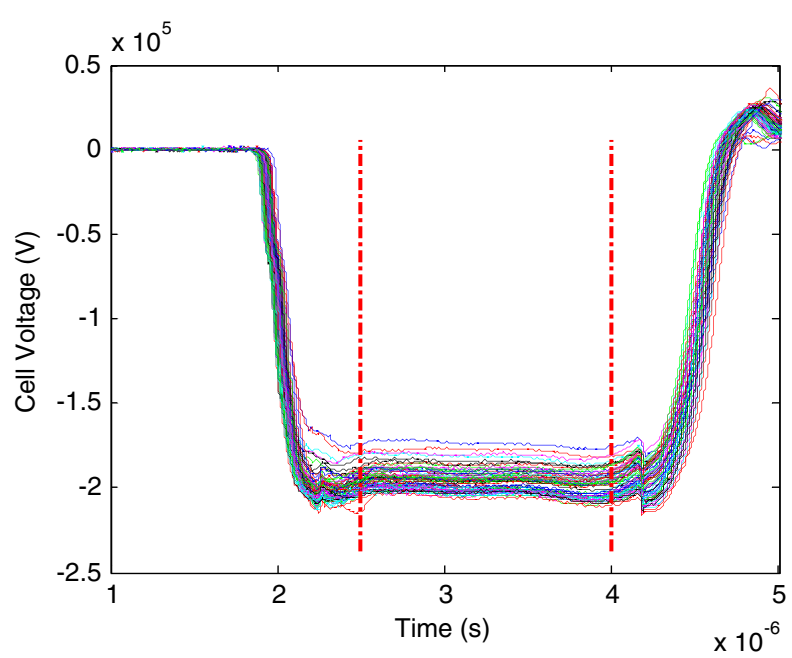

FIG. 12. Baseline cell voltages from shot 16729 taken with nominal zero timing. 


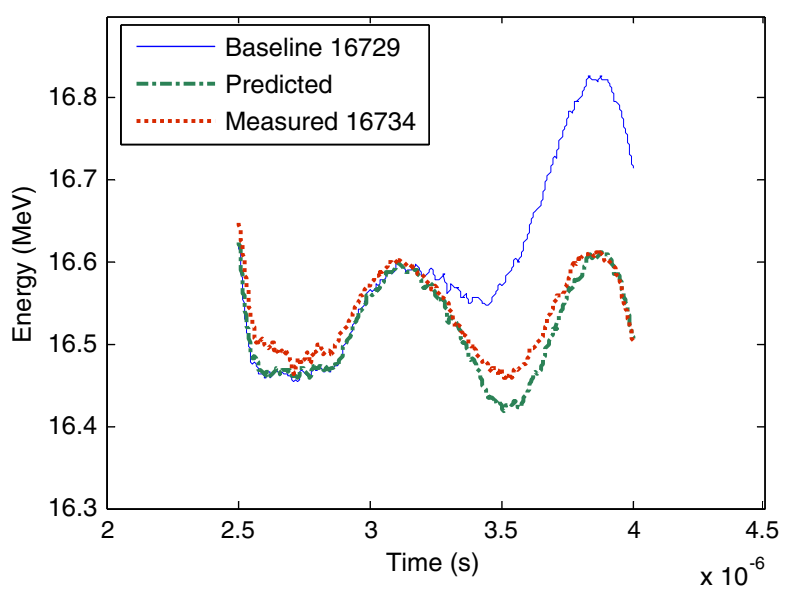

FIG. 13. Graphic showing loaded energy spread before adjustment $( \pm 1.126 \%)$, predicted $( \pm 0.622 \%)$, and measured results from $16734( \pm 0.571 \%)$. Measurement window is from 2.5 to $4.0 \mu \mathrm{s}$.

plotted for comparison. They are described in later sections. The analysis window could be slightly shifted, either left or right, or widened to alter the shape of the profile. For example, if the window is delayed slightly, and starts at $2.55 \mu \mathrm{s}$, and uses the same width, then the leading portion of the curve is reduced, and the trailing edge extends downward somewhat. However, axis-II operates with approximately this window, so we have used this analysis window for this work.

\section{B. Predicted}

The predicted energy spread is shown in Fig. 13. The optimizer values for the last three PFN/cells, 72, 73, and 74 , were $-101,-1000$, and $+100 \mathrm{~ns}$, respectively. Again the upstream PFN/cell times were kept fixed for this analysis and only the last three cell times were adjusted. The predicted energy spread is $\pm 0.622 \%$.

\section{Measured}

Using the timing adjustments described above, shot 16734 was executed. The actual, shifted-cell waveforms are shown in Fig. 14 along with cursors for the measurement window. The waveforms for injector voltage and beam current from 16734 were virtually identical to those shown in Figs. 10 and 11 for shot 16729 so are not repeated.

The energy spread for 16734 is shown in Fig. 13. Its spread of $\pm 0.571 \%$ is actually less than predicted and about $49 \%$ less than baseline. Its spread would be even smaller than calculated if not for the leading edge upward excursion. A slight delay in timing of the window would reduce that effect, but to be consistent, we have kept the window fixed. Given the fact that measured spread is better than predicted, there is more work to be done in improving

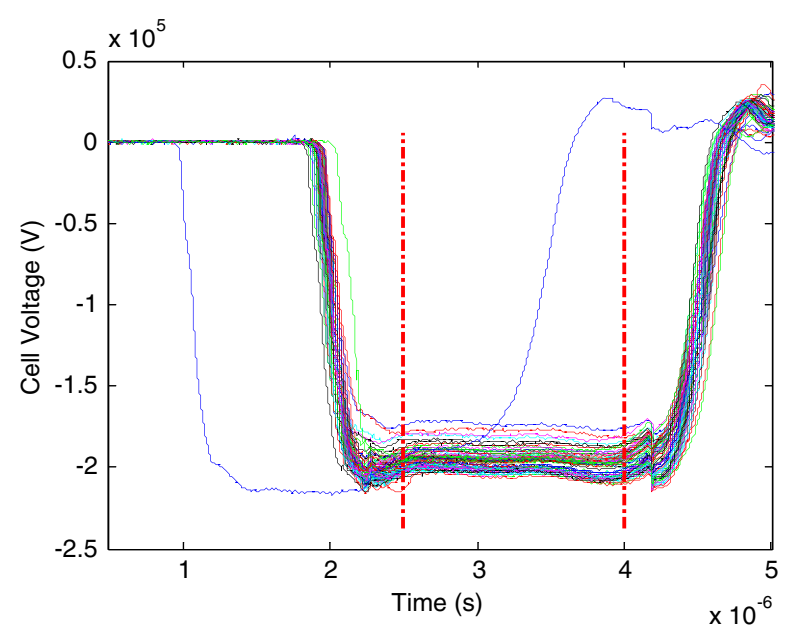

FIG. 14. Shot 16734 showing measured, loaded-cell voltages after optimization and time adjustments.

the models. Additionally, the sensitivity analysis for the loaded-cell case will be performed after the models are improved as it makes little sense to do that analysis until they are in closer agreement. Nevertheless, we expect the sensitivity of the loaded-cell energy spread to be similar to the unloaded results.

Because only the last three cells were time shifted to reduce the energy spread, there was modest reduction in beam motion at the end of the accelerator due to the fact that beam motion had already been induced upstream. What is significant is that the energy spread was reduced making tuning in the downstream transport easier allowing for better multipulse radiography, and that there was little or no additional induced beam motion. Experiments are continuing to measure improved spot size as a result of improved energy spread.

Obviously, we intend to continue to push the energy spread reduction further upstream such that beam motion can be reduced earlier in the accelerator. For instance, had the energy spread reduction been pushed further upstream, and assuming beam energy spread similar to the measured case shown in Fig. 13, then beam motion simulations performed in [22] suggest a reduction of beam sweep by as much as $50 \%$ or more.

Moreover, loaded-cell simulations, which have not yet been experimentally verified, show that if the last four trigger units (cells 69-74) are optimized, the energy spread is reduced to $\pm 0.403 \%$, and for the last six trigger units (cells $61-74$ ), the spread is $\pm 0.394 \%$, and for the last eight trigger units (cells 53-74), it is reduced to $\pm 0.389 \%$. These data indicate what appears to be a diminishing return as the improvement in energy spread reduction is decreasing as compared to the number of optimized cells. However, it is important to move the energy spread reduction as far upstream as practical to mitigate the effects of beam motion while taking into account focusing, steering, and other beam transport physics. 


\section{CONCLUSIONS}

This article described a nonlinear optimization technique which adjusts the timing of the cells on DARHT axis-II to improve multipulse radiography by minimizing beam-energy spread. It is a cost-effective technique that does not require extensive retuning of PFNs or cells, and can accommodate different operational cell voltage and beam parameters.

Baseline energy spread data, model predictions, and measurements for both unloaded and loaded-cell voltages were presented. The unloaded cell-voltage situation allows for simpler cell models, and cell voltages that maintain their voltage profile when advanced or retarded. For the loaded-cell simulations, the effects of cell loading due to beam current were synthesized in order for the optimizer to be able to adjust their timing. Cells that were not time shifted were fixed in time and empirical data were used for their voltage profiles.

Because of multiple local minima in the solution space, a random restart technique was used for the optimizer seed values. The output of the optimizer is a solution set containing timing adjustments for each of the user-selected trigger units.

To illustrate the performance of the optimization technique for unloaded cell voltages, a set of voltage waveforms from shot 14753 was acquired and a baseline energy spread determined. The optimizer was executed using the baseline data, and a solution set was generated which was used to adjust the timing of the trigger units. Two axis-II shots were then performed using timing offsets determined by the optimizer, and these results were presented in shots 14801 and 14802.

A Monte Carlo analysis was performed using Gaussian, 1- $\sigma$, jitter values distributed across the 74 cells to determine the sensitivity of the optimizer method. These jitter values showed the maximum-energy spread, essentially its degradation from predicted, based on what we attribute to be intrinsic system jitter. MC simulations were run using the 6-ns value which effectively set the limits for actual performance measurements. Both unloaded shots had measured energy spreads less than the MC-simulated worstcase energy spread.

Additionally, we examined the effects of beam loading on the cell voltages and showed that a significant reduction in energy spread can be accomplished by adjusting only the timing of the last three cells. We used only the last three cells to minimize risk to the accelerator while the models are being verified.

Future work is focused in several areas. One is to improve the accuracy of the loaded-cell-voltage synthesizer used in the optimizer. Another is to examine the benefits of energy spread reduction and concomitant beam motion reduction as a function of cell position. For example, significant beam motion reduction (and associated improvement in multipulse radiography) may be obtained by optimizing the timing of some group of cells upstream leaving others unaffected. And finally, work is proceeding to measure the sensitivity of the analysis, the improvement in spot size, and improve the computational effectiveness of the algorithms.

\section{ACKNOWLEDGMENTS}

The authors gratefully acknowledge the support and encouragement of Dr. Subrata Nath for this research. This work was supported by the U.S. National Nuclear Security Agency and the U.S. Department of Energy under Contract No. W-7405-ENG-36.

[1] S. Nath, in Proceedings of the XXV International Linac Conference, Tsukuba, Japan, 2010, pp. 750-754.

[2] C. Ekdahl et al., J. Korean Phys. Soc. 59, 3448 (2011).

[3] C. Ekdahl et al., in Proceedings of the XXIV Linear Accelerator Conference, Victoria, BC, Canada, 2008, pp. 311-313.

[4] M.J. Burns et al., in Proceedings of the 19th Particle Accelerator Conference, Chicago, Illinois, 2001 (IEEE, Piscataway, NJ, 2001), Vol. 1, p. 325.

[5] J. de Mascureau, CERN Accelerator School Report No. 79-94, 1994.

[6] H. Davis and R. Scarpetti, in Proceedings of the Linear Accelerator Conference, LINAC 2006, Knoxville, Tennessee, pp. 206-212.

[7] F. I. Burgess et al., in Proceedings of the 18th Particle Accelerator Conference, New York, 1999 (IEEE, New York, 1999), Vol. 5, p. 3257.

[8] Thomas P. Hughes, Harold Davis, Thomas C. Genoni, Mike Kang, and Benjamin A. Prichard, in Proceedings of the 21st IEEE Particle Accelerator Conference, Knoxville, Tennessee (IEEE, Piscataway, NJ, 2005), pp. 2269-2271.

[9] B. A. Prichard et al., in Proceedings of the 21st Particle Accelerator Conference, Knoxville, 2005 (IEEE, Piscataway, NJ, 2005), p. 169-173.

[10] K. Nielsen et al., in Proceedings of the 2007 Particle Accelerator Conference, Albuquerque, New Mexico (IEEE, New York, 2007), pp. 2385-2387.

[11] B. R. Poole and Y-J Chen, Report No. UCRL-JC-141921, 2001.

[12] S. Humphries, Jr., Principles of Charged Particle Acceleration (John Wiley and Sons, New York, 1999).

[13] E. Wadlinger, Los Alamos National Laboratory Report No. LA-UR-05-4656.

[14] W.L. Waldron and L. L. Reginato, in the Twenty-Fourth International Power Modulator Symposium, Norfolk, VA (IEEE, New York, 2000), pp. 179-182.

[15] M. Burns, H. Kirbie, T. McCuistian, K. Nielsen, H. Rutkowski, W. Waldron, S. Yu, E. Cook, and J. Watson, Twenty-fifth International Power Modulator Symposium (IEEE, Hollywood, CA, 2002), pp. 309-312.

[16] M. Schulze et al., in Proceedings of the 2007 Particle Accelerator Conference, Albuquerque, New Mexico (IEEE, New York, 2007), pp. 2627-2629. 
[17] E. G. Cook, B.S. Lee, S.A. Hawkins, E. M. Anaya, F. V. Allen, B.C. Hickman, J.S. Sullivan, and C. A. Brooksby, Pulsed Power Plasma Science (2001), Vol. 1, pp. 632-635.

[18] L. Wang, G. J. Caporaso, and E. G. Cook, in the $X X$ International Linac Conference, Monterey, CA, USA, 2000 (Stanford Linear Accelerator Center, Stanford, CA, 2000), pp. 509-511.
[19] Carl Ekdahl et al., IEEE Trans. Plasma Sci. 34 (2006) 460.

[20] Y-J. Chen, Nucl. Instrum. Methods Phys. Res. 292, 455 (1990).

[21] Mathworks, Inc., 3 Apple Hill Drive, Natick, MA 01761 [http://www.mathworks.com].

[22] Carl Ekdahl et al., Phys. Rev. ST Accel. Beams 14, 120401 (2011). 